\title{
SYNTHESIS OF SOME NOVEL (E)-METHYL 2,4-DIMETHYL-5-(3-OX0-3-PHENYLPROP-1-EN-1- YL)-1H-PYRROLE-3-CARBOXYLATE DERIVATIVES AS ANTIMICROBIAL AGENT
}

\author{
MAHESH HUBLIKAR ${ }^{1}$, PRASHANT DIXIT ${ }^{2}$, VIKAS KADU ${ }^{1}$, SACHIN SHIRAME ${ }^{1}$, DATTATRAYA RAUT ${ }^{1}$, \\ RAGHUNATH BHOSALE ${ }^{*}$, SHRAVAN JADHAV ${ }^{3}$
}

\begin{abstract}
${ }^{1}$ Organic Chemistry Research Laboratory, School of Chemical Sciences, Solapur University, Solapur, Maharashtra, India. ${ }^{2}$ Department of Microbiology, Dr. Babasaheb Ambedkar Marathwada University, Sub Campus, Osmanabad, Maharashtra, India. ${ }^{3}$ Department of Chemistry, DBF Dayanand College of Arts and Science, Solapur, Maharashtra, India. Email: bhosale62@yahoo.com
\end{abstract}

Received: 15 October 2018, Revised and Accepted: 01 December 2018

ABSTRACT

Objective: The objective of the present study was to synthesize a series of some novel ( $E$ )-methyl 2,4-dimethyl-5-(3-oxo-3-phenylprop-1-en-1-yl)-1Hpyrrole-3-carboxylate derivatives and to evaluate it's in vitro antimicrobial activities.

Methods: A novel series of (E)-methyl 2,4-dimethyl-5-(3-oxo-3-phenylprop-1-en-1-yl)-1H-pyrrole-3-carboxylate derivative (8a-l) has been synthesized by cyclization (Knorr reaction) hydrolysis, decarboxylation, and Vilsmeier-Haack formylation reaction. 5-formyl-2,4-dimethyl-1Hpyrrole-3-carboxylate 6 undergo condensation with acetophenone derivatives 7a-l in methanol and potassium hydroxide. The synthesized compounds were screened for in vitro antimicrobial screening.

Results: The structures of the synthesized compounds were characterized by infrared, $1 \mathrm{H}$ nuclear magnetic resonance, and mass spectroscopy. The antimicrobial activity data revealed that the synthesized derivatives possess good antibacterial and antifungal activity which is attributed due to the presence of the heterocyclic ring; further, the activity increased with the introduction of a methoxy group in the structure.

Conclusions: New pyrrole chalcone derivatives act as significant antimicrobial agents, easy work-up procedure and reaction take place with minimum side product. Antimicrobial activity report provides an interesting template for the syntheses of new antimicrobial agents and may be helpful for the design of new therapeutic tools.

Keywords: Antimicrobial, Gram-positive, Gram-negative bacteria, Pyrrole chalcones.

(C) 2019 The Authors. Published by Innovare Academic Sciences Pvt Ltd. This is an open access article under the CC BY license (http://creativecommons. org/licenses/by/4. 0/) DOI: http://dx.doi.org/10.22159/ajpcr.2019.v12i2.30275

\section{INTRODUCTION}

Heterocyclic compounds have a wide range of applications in pharmaceutical chemistry. Heterocyclic compounds have natural as well as synthetic source. Pyrrole has gain remarkable importance among the new heterocycles due to biological potential. Pyrrole has antimalarial and enzyme inhibition properties. Pyrrole is five-membered nitrogencontaining heterocyclic compound. Colorless liquid compound and on exposing to air it becomes darken. It has importance in more complex macromolecules such as porphyrins of heme and chlorophyll. It was isolated from pyrolysate of bone. Its name came from Greek [1]. In recent years, there has been growing interest in chalcones and their important role in the prevention of various diseases such as cancer and chronic inflammation.

The chalcones are structurally simple and largest class of plant secondary metabolites, which serves as a defense mechanism in plants to counteract reactive oxygen species to prevent damage by micro-organisms [2]. Antimicrobial activity of bis-heterocyclic chalcones was tested against selective pathogens Escherichia coli, Candida albicans, and Aspergillus niger [3]. Several studies have pointed out the antimicrobial potential of chalcones against a wide range of fungi and bacteria, including resistant ones, clearly indicating that they are attractive target compounds for the discovery and development of new anti-infective agents [4-10]. These flavonoid family compounds display an impressive area of biological activities, among which antibacterial [11], antifungal [12], antioxidant [13,14], antileishmanial [15], antimalarial [16], angiogenesis inhibitor [17], antiinflammatory [18,19], antiviral [20], antimitotic [21], anticancer [22], anti-invasive [23,24], and antiproliferative [25]. Activities have been cited in the literature. Chalcones strongly inhibit the polymerization of tubulin by binding to the colchicine-binding site [16]. The $\alpha$, $\beta$-unsaturated compounds may possess diverse pharmacological activities, and it has been found that the synthesized chalcones interact with the bovine serum albumin, a protein mainly responsible for the transportation of a number of compounds [26]. Substantial preliminary antibacterial activity was reported for the new chalcones at C8 of the coumarin nucleus, against Gram-positive and a Gram-negative bacteria [27]. In view of the pharmacological activities of phenothiazine, pyrazole, and pyrrole a number of the 2-substituted, pyrrole derivatives have been synthesized which contain pyrrole heterocycles [28].

Therefore, in view of these important biological activities of pyrrole scaffold. We reported the synthesis of some novel (E)-methyl 2,4-dimethyl-5-(3-oxo-3-phenylprop-1-en-1-yl)-1H-pyrrole-3carboxylate derivatives and tested the antibacterial and antifungal activities of the new resulting compounds which have been found to possess an interesting profile of pharmacological activity, against Gram-negative, Gram-positive bacteria and fungi 8f, 8e, 8c. Chemically chalcones are open chain flavonoids in which the two aromatic rings are joined together by three carbons, $\alpha$, and $\beta$-unsaturated carbonyl system. Fundamentally, they are considered as derivatives of phenyl styryl ketone. In view of the varied biological and pharmacological applications, we have synthesized some new $(E)$-methyl 2,4-dimethyl-5(3-oxo-3-phenylprop-1-en-1-yl)-1H-pyrrole-3-carboxylate derivatives. In general, chalcones contain C6-C3-C6 unit but the compounds reported in the present study contain $\mathrm{C} 6-\mathrm{C} 3-\mathrm{C} 5$ unit. 


\section{EXPERIMENTAL}

\section{METHODS}

The melting points of all the synthesized compounds were determined in open capillary tubes and are uncorrected. The purity of all compounds was checked by thin-layer chromatography (TLC) Silica gel $60 \mathrm{~F}_{254}$. Infrared (IR) spectra were recorded on Fourier-transform IR (ModelNicolet iS-10 Mid) ${ }^{1} \mathrm{H}$ NMR spectra were recorded on $400 \mathrm{MHz}$-NMR Spectrometer $\mathrm{CDCl}_{3} /$ dimethyl sulfoxide (DMSO)- $d_{6}$ (Bruker Biospin International $\mathrm{AG}$ ); chemical shifts $(\delta)$ are in parts per million (ppm) relative to TMS and coupling constant $($ ) is expressed in hertz (Hz). Mass spectra were recorded on a Thermo Scientific Q-Exactive, Accela 1250 pump, Macro mass spectrometer (Water, Milford, MA) by electrospray method (ES). TLC was performed on the and visualized in UV light. All chemicals and solvents were obtained from Spectrochem, Aldrich, or Merck and are of analytical grade, and were used freshly after opening.

Synthesis of diethyl 3, 5-dimethyl-1H-pyrrole-2,4-dicarboxylate (2) To prepare diethyl 3, 5-dimethyl-1H-pyrrole-2,4-dicarboxylate, a mixture of acetic acid $(40 \mathrm{ml})$ and ethyl acetoacetic ester $1(20 \mathrm{ml}, 156 \mathrm{mmol})$ was added an aqueous solution of sodium nitrite $(5.6 \mathrm{~g}, 81 \mathrm{mmol})$ in water $(6 \mathrm{ml})$ cooled in an ice bath to keep the temperature of reaction mixture $5-10^{\circ} \mathrm{C}$. The yellowish clear reaction mixture was kept for another $2.5 \mathrm{~h}$ at the same temperature to further complete reaction. Then, zinc powder ( $11 \mathrm{~g}, 52.34 \mathrm{mmol}, 300 \mathrm{mesh}$ ) was added to this mixture portion wise while the reaction mixture was kept $<25^{\circ} \mathrm{C}$. It was then heated to $45-50^{\circ} \mathrm{C}$ for $10 \mathrm{~min}$ then continued slow heating to $95^{\circ} \mathrm{C}$ for another $1 \mathrm{~h}$ exotherm was observed. Cooled reaction mass to room temperature, the yellowish precipitate was collected and washed with ice water to remove the acetic acid. Recrystallized the yellow crude solid from ethanol to afford a pale yellow crystalline solid powder of pure 2 (35 g, yield 93\%), mp. (melting point) $137-138^{\circ} \mathrm{C}$. lit. mp. $135-136^{\circ} \mathrm{C}[29,30] .{ }^{1} \mathrm{H}$ NMR $\left(400 \mathrm{MHz}, \mathrm{CDCl}_{3}\right) \delta 1.35-1.39$ $\left(\mathrm{m}, 6 \mathrm{H}, 2 \mathrm{X} \mathrm{CH}_{3}\right), 2.52\left(\mathrm{~s}, 3 \mathrm{H}, \mathrm{CH}_{3}\right), 2.57\left(\mathrm{~s}, 3 \mathrm{H}, \mathrm{CH}_{3}\right), 4.27-4.36(\mathrm{~m}, 4 \mathrm{H}, 2$ $\left.\mathrm{X} \mathrm{CH}_{2}\right), 8.90(\mathrm{~s}, 1 \mathrm{H},-\mathrm{NH}$ pyrrole).

\section{Synthesis of 4-(ethoxycarbonyl)-3,5-dimethyl-1H-pyrrole-2-} carboxylic acid (3)

To prepare 4-(ethoxycarbonyl)-3,5-dimethyl-1H-pyrrole-2-carboxylic acid, compound $2(20 \mathrm{~g}, 83.6 \mathrm{mmol})$ was added into a flask equipped with a stirrer, a thermometer, and a condenser; absolute ethanol $(120 \mathrm{ml})$ was added into the flask. An aqueous solution $(240 \mathrm{ml})$ of potassium hydroxide (11.8 g, $210.3 \mathrm{mmol}$ ) was added heated at $50-60^{\circ} \mathrm{C}$ for $10 \mathrm{~h}$ with monitoring by TLC until reaction completion. The reaction was then stopped by cooling down to room temperature. The reaction mixture was poured into ice-water $(600 \mathrm{ml})$ to form an aqueous solution. A small amount of insoluble substance was filtered and kept aside. Aqueous layers were adjusted with hydrochloride $(2 \mathrm{~mol} / \mathrm{L})$ to $\mathrm{pH} 4$ to afford some white precipitates, which were collected through filtering and washing with ice-water to provide 3 (15 g, yield $85 \%)$, m. p. $169-170^{\circ} \mathrm{C}$ (lit. $235-236^{\circ} \mathrm{C}$ [30] (eluent: chloroform/methanol, $\mathrm{v} / \mathrm{v}=10: 1)$.

\section{Synthesis of 2, 4-dimethyl-1H-pyrrole-3-carboxylic acid (4)}

To prepare 2,4-dimethyl-1H-pyrrole-3-carboxylic acid, compound $3(10 \mathrm{~g}, 47.35 \mathrm{mmol})$ was added into flask equipped with a stirrer, a thermometer, and condenser; $\mathrm{HCl}(5 \mathrm{ml})$ and methanol $(150 \mathrm{ml})$ were added into the flask and heated up to $75-80^{\circ} \mathrm{C}$ for $1.5 \mathrm{~h}$ with monitoring by TLC until completion of decarboxylation. The reaction was cooled down to room temperature, further cooled up to $0-5^{\circ} \mathrm{C}$. Slowly added solution of $\mathrm{KOH}(8.8 \mathrm{~g})$ dissolving in water $(100 \mathrm{ml})$ and the reaction mass was heated at $60-65^{\circ} \mathrm{C}$ temperature for 5-6 $\mathrm{h}$ with monitoring by TLC until completion of hydrolysis. Stopped heating and cooled to room temperature. The reaction mass was extracted with ethyl acetate to remove impurities and separated aqueous layer was collected and acidified with 1:1 $\mathrm{HCl}$ to $\mathrm{pH} \mathrm{3-4} \mathrm{to} \mathrm{afford} \mathrm{light} \mathrm{brown} \mathrm{solid} \mathrm{precipitates,}$ which was collected through filtering and washing with ice-water to provide compound 4 ( 5.5 g, yield $83 \%$ ), m. p. $75-76^{\circ} \mathrm{C}$ (lit. $74-76^{\circ} \mathrm{C}[30]$ (eluent: chloroform/methanol, $\mathrm{v} / \mathrm{v}=10: 1$ ).
Synthesis of methyl 2,4-dimethyl-1H-pyrrole-3-carboxylate (5) To prepare methyl 2,4-dimethyl-1H-pyrrole-3-carboxylate, compound $4(5.0 \mathrm{~g}, 35 \mathrm{mmol})$ was added into flask equipped with a stirrer, a thermometer, and condenser; $\mathrm{H}_{2} \mathrm{SO}_{4}(0.2 \mathrm{ml})$ and methanol $(75 \mathrm{ml})$ into the flask and heated up to $60-65^{\circ} \mathrm{C}$ for $1.5 \mathrm{~h}$ monitored by TLC until reaction completion. The reaction was then stopped by cooling down to room temperature, further cooled up to $0-5^{\circ} \mathrm{C}$. The reaction mixture was poured into ice-water $(100 \mathrm{ml})$ to afford light brown solid precipitates, which was collected through filtering and washing with ice-water to provide $5(4.12 \mathrm{~g}, 68 \%), \mathrm{m} . \mathrm{p} .70-72^{\circ} \mathrm{C}$ (eluent: chloroform/ methanol, $\mathrm{v} / \mathrm{v}=10: 1$ )

\section{Synthesis of methyl 5-formyl-2,4-dimethyl-1H-pyrrole-3-} carboxylate (6)

Anhydrous DMF (2.5 ml, $200 \mathrm{mmol})$ in a dry flask was precooled to $0^{\circ} \mathrm{C}$ with a salt-ice bath, into which a solution of $\mathrm{POCl}_{3}(3.60 \mathrm{ml}, 320 \mathrm{mmol})$ in anhydrous dichloromethane $(10 \mathrm{ml})$ was added dropwise. The temperature should be kept $<3^{\circ} \mathrm{C}$ during the whole addition process. The reaction mixture was stirred for $0.5 \mathrm{~h}$ using an ice-bath before dropwise addition of a solution of methyl 2,4-dimethyl-1H-pyrrole-3-carboxylate $5(4.0,26.11 \mathrm{mmol})$ in anhydrous dichloromethane $(20.0 \mathrm{ml})$. The mixture was heated under reflux for $3 \mathrm{~h}$ and monitored by TLC until reaction completion. The reaction was then stopped by cooling down to room temperature, further cooled up to $0-5^{\circ} \mathrm{C}$. Neutralize the reaction mixture using $30 \%$ aq. (Aqueous) potassium carbonate solution till $\mathrm{pH}$ 8-9. Stirred the reaction mass for $1 \mathrm{~h}$ at room temperature, separated the organic layer using separating funnel, extracted the aqueous layer with DCM $(100 \mathrm{ml} \times 3$ times $)$ separated organic layer was washed with water $(50 \mathrm{ml} \times 3$ times) and separated organic layer was dried over sodium sulfate, distilled out DCM under vacuum completely to afford pale-gray solid, dried under vacuum to afford compound 6 (4.5 g, yield $95 \%$ ), m. p. $167-169^{\circ} \mathrm{C}$ (eluent: chloroform/methanol, $\mathrm{v} / \mathrm{v}=10: 1)$. IR $(\mathrm{KBr}) \mathrm{cm}^{-1} 3244(-\mathrm{NH}), 1698(\mathrm{C}=0), 1559(\mathrm{C}=\mathrm{C}),{ }^{1} \mathrm{H}$ $\operatorname{NMR}\left(400 \mathrm{MHz}, \mathrm{CDCl}_{3}\right) \delta 2.569-2.592\left(\mathrm{~s}, 6 \mathrm{H},-\mathrm{CH}_{3}\right), 3.863\left(\mathrm{~s}, 3 \mathrm{H},-\mathrm{OCH}_{3}\right)$, 9.637 (s, 1H, - CHO), 9.932 (s, 1H, NH).

General procedure for the synthesis of $(E)$-methyl 2,4-dimethyl-5(3-oxo-3-phenylprop-1-en-1-yl)-1H-pyrrole-3-carboxylate (8a-i) An aqueous solution of $\mathrm{KOH}(0.093 \mathrm{~g}, 1.65 \mathrm{mmol})$ was added to cooled suspension of 1-(4-fluorophenyl) ethanone 7a $(0.190 \mathrm{~g}, 1.38 \mathrm{mmol})$ and methanol-water mixture $5 \mathrm{ml}$ (9.5:0.5), this mixture was stirred $5-10^{\circ} \mathrm{C}$ for $30 \mathrm{~min}$. After $30 \mathrm{~min}$ added methyl 5-formyl-2,4-dimethyl$1 \mathrm{H}$-pyrrole-3-carboxylate $6(0.250 \mathrm{~g}, 1.38 \mathrm{~mol})$. Raised temperature up to room temperature and the mixture was stirred at room temperature for overnight. The reaction mixture was poured into water. The solid product (8a) separated out was filtered off and washed with plenty of water finally crystallized from ethanol. The compounds (8a-i) were prepared by following the aforementioned procedure. Their structures have been confirmed by mass, IR, and ${ }^{1} \mathrm{H}$ NMR spectra.

(E)-methyl 5-(3-(4-fluorophenyl)-3-oxoprop-1-en-1-yl)-2,4-dimethyl1H-pyrrole-3- carboxylate (8a)

Yield: 75\%; molecular formula (MF): $\mathrm{C}_{17} \mathrm{H}_{16} \mathrm{FNO}_{3} / 301.31$; yellow solid; MP. 207-210요 IR (KBr) cm ${ }^{-1}: 3235(-\mathrm{NH}), 1696$ (C=0), 1530 (C = C); ${ }^{1} \mathrm{H}$ NMR $\left(400 \mathrm{MHz}, \mathrm{DMSO}-\mathrm{d}_{6}\right) \delta 2.396\left(\mathrm{~s}, 3 \mathrm{H},-\mathrm{CH}_{3}\right), 2.574\left(\mathrm{~s}, 3 \mathrm{H},-\mathrm{CH}_{3}\right)$, $3.838\left(\mathrm{~s}, 3 \mathrm{H},-\mathrm{OCH}_{3}\right), 6.978-7.016\left(\mathrm{~d}, J=15.2 \mathrm{H}_{\mathrm{z}}, 1 \mathrm{H}\right.$, ethylenic $)$, 7.100-7.143 (m, 2H, -Ar), 7.789-7.828 (d, $J=15.6 \mathrm{H}_{\mathrm{z}}, 1 \mathrm{H}$, ethylenic), 7.978-8.014 (m, 2H, -Ar), 9.0159 (s,1H, -NH); HRMS = $302.1187[\mathrm{M}+\mathrm{H}]$.

(E)-methyl 2,4-dimethyl-5-(3-oxo-3-(3,4,5-trimethoxyphenyl) prop1-en-1-yl)-1H-pyrrole-3-carboxylate (8b)

Yield: 79\%; MF: $\mathrm{C}_{20} \mathrm{H}_{23} \mathrm{NO}_{6} / 373.4$; yellow solid; MP. $138-141^{\circ} \mathrm{C}$; IR $(\mathrm{KBr}) \mathrm{cm}^{-1}$ : $3379(-\mathrm{NH}), 1675(\mathrm{C}=0), 1545(\mathrm{C}=\mathrm{C}) ;{ }^{1} \mathrm{H}$ NMR $(400 \mathrm{MHz}$, DMSO- $\left.d_{6}\right) \delta 2.412\left(\mathrm{~s}, 3 \mathrm{H},-\mathrm{CH}_{3}\right), 2.588\left(\mathrm{~s}, 3 \mathrm{H},-\mathrm{CH}_{3}\right), 3.842\left(\mathrm{~s}, 3 \mathrm{H},-\mathrm{OCH}_{3}\right)$, 3.915-3.929 (s, 9H, $\left.-\mathrm{OCH}_{3}\right), 6.920-6.958\left(\mathrm{~d}, J=15.2 \mathrm{H}_{\mathrm{z}}, 1 \mathrm{H}\right.$, ethylenic); 7.199-7.216 (d, 2H, -Ar); 7.794-7.833 (d, $J=15.6 \mathrm{H}_{\mathrm{Z}^{\prime}}, 1 \mathrm{H}$, ethylenic); $8.939(\mathrm{~s}, 1 \mathrm{H},-\mathrm{NH}) ; \mathrm{HRMS}=374.1598[\mathrm{M}+\mathrm{H}]$. 
(E)-methyl 5-(3-(4-cyanophenyl)-3-oxoprop-1-en-1-yl)-2,4-dimethyl1 H-pyrrole-3-carboxylate $(8 \mathrm{c})$

Yield: 69\%; MF: $\mathrm{C}_{18} \mathrm{H}_{16} \mathrm{~N}_{2} \mathrm{O}_{3} / 308.33$; yellow solid; MP.218-220 ${ }^{\circ} \mathrm{C}$; $\operatorname{IR}(\mathrm{KBr}) \mathrm{cm}^{-1}$ : $3338(-\mathrm{NH}), 2235$ (CN), 1698 (C=0), 1555 (C=C); ${ }^{1} \mathrm{H}$ NMR $\left(400 \mathrm{MHz}, \mathrm{DMSO}-d_{6}\right) \delta 2.368\left(\mathrm{~s}, 3 \mathrm{H},-\mathrm{CH}_{3}\right), 2.529\left(\mathrm{~s}, 3 \mathrm{H},-\mathrm{CH}_{3}\right)$, $3.791\left(\mathrm{~s}, 3 \mathrm{H},-\mathrm{OCH}_{3}\right), 7.243-7.81\left(\mathrm{~d}, J=15.2 \mathrm{H}_{\mathrm{z}} 1 \mathrm{H}\right.$, ethylenic), 7.732$7.753(\mathrm{~d}, 2 \mathrm{H},-\mathrm{Ar}), 7.765-7.804\left(\mathrm{~d}, J=15.6 \mathrm{H}_{\mathrm{z}} 1 \mathrm{H}\right.$, ethylenic), 8.083$8.104(\mathrm{~d}, 2 \mathrm{H},-\mathrm{Ar}) 10.862(\mathrm{~s}, 1 \mathrm{H},-\mathrm{NH})$; HRMS = $309.1234[\mathrm{M}+\mathrm{H}]$.

(E)-methyl 2,4-dimethyl-5-(3-(4-nitrophenyl)-3-oxoprop-1-en-1yl)-1H-pyrrole-3-carboxylate (8d)

Yield: $71 \%$; $M F$ : $\mathrm{C}_{17} \mathrm{H}_{16} \mathrm{~N}_{2} \mathrm{O}_{5} / 328.32$; orange solid; MP. 99-101 ${ }^{\circ} \mathrm{C}$; IR $(\mathrm{KBr}) \mathrm{cm}^{-1}: 3218(-\mathrm{NH}), 1687(\mathrm{C}=0), 1558(\mathrm{C}=\mathrm{C}) ;{ }^{1} \mathrm{H}$ NMR $(400 \mathrm{MHz}$, DMSO- $\left.d_{6}\right) \delta 2.418\left(\mathrm{~s}, 3 \mathrm{H},-\mathrm{CH}_{3}\right), 2.598\left(\mathrm{~s}, 3 \mathrm{H},-\mathrm{CH}_{3}\right), 3.848\left(\mathrm{~s}, 3 \mathrm{H},-\mathrm{OCH}_{3}\right)$, 6.926-6.964 (d, $J=15.2 \mathrm{H}_{\mathrm{z}}, 1 \mathrm{H}$, ethylenic), $7.821-7.859\left(\mathrm{~d}, J=15.2 \mathrm{H}_{\mathrm{z}^{\prime}}\right.$ $1 \mathrm{H}$, ethylenic), 8.082-8.104 (d, J=8.8Hz, $2 \mathrm{H},-\mathrm{Ar}) ; 8.300-8.322(\mathrm{~d}, J=8.8$, $2 \mathrm{H},-\mathrm{Ar}), 8.952(\mathrm{~s}, 1 \mathrm{H},-\mathrm{NH}) ; \mathrm{HRMS}=329.1132[\mathrm{M}+\mathrm{H}]$.

(E)-methyl 5-(3-(4-chlorophenyl)-3-oxoprop-1-en-1-yl)-2,4-dimethyl1 H-pyrrole-3-carboxylate (8e)

Yield: 72\%; MF: $\mathrm{C}_{17} \mathrm{H}_{16} \mathrm{ClNO}_{3} / 317.76$; brownish yellow solid; MP. 170-172 ${ }^{\circ} \mathrm{C}$; IR(KBr) cm ${ }^{-1}: 3258(-\mathrm{NH}), 1697$ (C=0), 1530 (C=C); ${ }^{1} \mathrm{H}$ NMR (400 MHz, DMSO- $\left.d_{6}\right) \delta 2.4\left(\mathrm{~s}, 3 \mathrm{H},-\mathrm{CH}_{3}\right), 2.6\left(\mathrm{~s}, 3 \mathrm{H},-\mathrm{CH}_{3}\right), 3.839$ (s, $3 \mathrm{H},-\mathrm{OCH}_{3}$ ), 6.954-6.992 (d, $J=15.2 \mathrm{H}_{\mathrm{z}} 1 \mathrm{H}$, ethylenic), 7.405-7.426 (d, $J=8.4 \mathrm{H}_{\mathrm{z}} 2 \mathrm{H},-\mathrm{Ar}$ ), 7.789-7.827 (d, $J=15.2 \mathrm{H}_{\mathrm{z}} 1 \mathrm{H}$, ethylenic), 7.909$7.888\left(\mathrm{~d}, J=8.4 \mathrm{H}_{\mathrm{z}} 2 \mathrm{H},-\mathrm{Ar}\right), 9.047(\mathrm{~s}, 1 \mathrm{H},-\mathrm{NH}) ;$ ESI-MS $=318.0903$ $[\mathrm{M}+\mathrm{H}]$.

(E)-methyl 5-(3-(4-methoxyphenyl)-3-oxoprop-1-en-1-yl)-2,4-dimethyl1H-pyrrole-3-carboxylate (8f)

Yield: 67\%; MF: $\mathrm{C}_{18} \mathrm{H}_{19} \mathrm{NO}_{4} / 313.34$; yellow solid; MP.179-180 ${ }^{\circ} \mathrm{C}$; $\operatorname{IR}(\mathrm{KBr}) \mathrm{cm}^{-1}: 3191(-\mathrm{NH}), 1699(\mathrm{C}=0), 1534(\mathrm{C}=\mathrm{C}) ;{ }^{1} \mathrm{H}$ NMR $(400 \mathrm{MHz}$, DMSO- $\left.d_{6}\right) \delta 2.396\left(\mathrm{~s}, 3 \mathrm{H},-\mathrm{CH}_{3}\right), 2.570\left(\mathrm{~s}, 3 \mathrm{H},-\mathrm{CH}_{3}\right), 3.849\left(\mathrm{~s}, 3 \mathrm{H},-\mathrm{OCH}_{3}\right)$, 3.882 (s, $3 \mathrm{H},-\mathrm{OCH}_{3}$ ); 6.904-6.925 (d, $\left.J=8.4 \mathrm{H}_{\mathrm{z}} 2 \mathrm{H},-\mathrm{Ar}\right), 7.041-7.080$ (d, $J=15.6 \mathrm{H}_{\mathrm{z}} 1 \mathrm{H}$, ethylenic), 7.787-7.826 (d, $J=15.6 \mathrm{H}_{\mathrm{z}} 1 \mathrm{H}$, ethylenic), 7.969-7.990 (d, J= 8.4 H 2 $2 \mathrm{H},-\mathrm{Ar}), 9.106(\mathrm{~s}, 1 \mathrm{H}, \mathrm{NH})$; HRMS = 314.1379 $[\mathrm{M}+\mathrm{H}]$.
(E)-methyl 5-(3-(2,4-dimethoxyphenyl)-3-oxoprop-1-en-1-yl)-2,4dimethyl-1H-pyrrole-3-carboxylate (8g)

Yield: 65\%; MF: $\mathrm{C}_{19} \mathrm{H}_{21} \mathrm{NO}_{5} / 343.37$; yellow solid; MP.137-139 ${ }^{\circ} \mathrm{C}$; IR(KBr) cm ${ }^{-1}: 3225(-\mathrm{NH}), 1693(\mathrm{C}=0), 1536(\mathrm{C}=\mathrm{C}) ;{ }^{1} \mathrm{H}$ NMR $(400$ $\left.\mathrm{MHz}, \mathrm{DMSO}-\mathrm{d}_{6}\right) \delta 2.375\left(\mathrm{~s}, 3 \mathrm{H},-\mathrm{CH}_{3}\right), 2.558\left(\mathrm{~s}, 3 \mathrm{H},-\mathrm{CH}_{3}\right) ; 3.865(\mathrm{~s}$, $\left.9 \mathrm{H},-\mathrm{OCH}_{3}\right) ; 6.477-6.483(\mathrm{~d}, 1 \mathrm{H},-\mathrm{Ar}), 6.536-6.558\left(\mathrm{~d}, J=8.8 \mathrm{H}_{\mathrm{z}} 1 \mathrm{H},-\mathrm{Ar}\right)$, 6.942-6.981 (d, $J=15.6 \mathrm{H}_{\mathrm{z}} 1 \mathrm{H}$, ethylenic), 7.637-.637 (d, $J=15.6 \mathrm{~Hz}$ $1 \mathrm{H}$, ethylenic), 7.680-7.701 (d, $\left.J=8.4 \mathrm{H}_{\mathrm{z}} 1 \mathrm{H},-\mathrm{Ar}\right), 8.812(\mathrm{~s}, 1 \mathrm{H},-\mathrm{NH})$; ESI-MS: $344.20[\mathrm{M}+\mathrm{H}]$.

(E)-methyl 2,4-dimethyl-5-(3-oxo-3-phenylprop-1-en-1-yl)-1H-pyrrole3-carboxylate (8h)

Yield: 61\%; $\mathrm{MF}: \mathrm{C}_{17} \mathrm{H}_{17} \mathrm{NO}_{3} / 283.32$; yellow solid; MP. $150-153^{\circ} \mathrm{C}$; $\mathrm{IR}(\mathrm{KBr})$ $\mathrm{cm}^{-1}: 3222(-\mathrm{NH}), 1698(\mathrm{C}=0), 1540(\mathrm{C}=\mathrm{C}) ;{ }^{1} \mathrm{H}$ NMR (400 MHz, DMSO- $\left.d_{6}\right)$ $\delta 2.401\left(\mathrm{~s}, 3 \mathrm{H},-\mathrm{CH}_{3}\right) ; 2.575\left(\mathrm{~s}, 3 \mathrm{H},-\mathrm{CH}_{3}\right), 3.837\left(\mathrm{~s}, 3 \mathrm{H},-\mathrm{OCH}_{3}\right), 7.017-$ 7.056 (d, $J=15.6 \mathrm{~Hz} 1 \mathrm{H}$, ethylenic), 7.435-7.473 (m, 2H, -Ar), 7.5267.566 (m, 1H, -Ar), 7.796-7.834 (d, $J=15.2 \mathrm{H}_{\mathrm{z}} 1 \mathrm{H}$, ethylenic), 7.956$7.976(\mathrm{~d}, J=8 \mathrm{~Hz} 2 \mathrm{H}-\mathrm{Ar}), 9.052$ (s, 1H, -NH); ESI-MS:284.2000 [M+H].

(E)-methyl 2, 4-dimethyl-5-(3-oxo-3-(p-tolyl)prop-1-en-1-yl)-1Hpyrrole-3-carboxylate (8i)

Yield: 59\%; MF: $\mathrm{C}_{18} \mathrm{H}_{19} \mathrm{NO}_{3} / 297.34$; yellow solid; MP.178- $181^{\circ} \mathrm{C}$; IR(KBr) cm ${ }^{-1}: 3195$ (-NH), 2985 (-CH), 1693 (C=0), 1539 (C=C); ${ }^{1} \mathrm{H}$ NMR $\left(400 \mathrm{MHz}, \mathrm{DMSO}-d_{6}\right) \delta 2.404\left(\mathrm{~s}, 6 \mathrm{H},-\mathrm{CH}_{3}\right), 2.563\left(\mathrm{~s}, 3 \mathrm{H},-\mathrm{CH}_{3}\right), 3.831(\mathrm{~s}$, $\left.3 \mathrm{H},-\mathrm{OCH}_{3}\right), 7.056-7.095(\mathrm{~d}, J=15.6 \mathrm{~Hz} 1 \mathrm{H}$, ethylenic), 7.212-7.232 (d, $J$ $=8 \mathrm{~Hz} 2 \mathrm{H}-\mathrm{Ar}$ ), 7.784-7.823 (d, $J=15.6 \mathrm{~Hz} 1 \mathrm{H}$, ethylenic), 7.861-7.881 (d, J = $8 \mathrm{~Hz} 2 \mathrm{H},-\mathrm{Ar}$ ), 9.269 (s,1H, -NH); ESI-MS: 298.20 [M+H].

(E)-methyl 5-(3-(2,5-dimethoxyphenyl)-3-oxoprop-1-en-1-yl)-2,4dimethyl-1H-pyrrole-3-carboxylate (8j)

Yield: $60 \%$; $\mathrm{MF}: \mathrm{C}_{19} \mathrm{H}_{21} \mathrm{NO}_{5} / 343.37$; yellow solid; $\mathrm{MP} .122-124^{\circ} \mathrm{C}$; $\mathrm{IR}(\mathrm{KBr}) \mathrm{cm}^{-1}: 3252(-\mathrm{NH}), 1682(\mathrm{C}=\mathrm{O}), 1546(\mathrm{C}=\mathrm{C}) ;{ }^{1} \mathrm{H}$ NMR $(400 \mathrm{MHz}$, DMSO- $\left.d_{6}\right) \delta 2.356\left(\mathrm{~s}, 3 \mathrm{H},-\mathrm{CH}_{3}\right), 2.552\left(\mathrm{~s}, 3 \mathrm{H},-\mathrm{CH}_{3}\right), 3.825\left(\mathrm{~s}, 9 \mathrm{H},-\mathrm{OCH}_{3}\right)$, 6.860-6.899 (d, $J=15.61 \mathrm{HZ} 1 \mathrm{H}$, ethylenic), 6.904-7.008 (m, 2H -Ar), 7.121-7.128 (d, 1H, -Ar), 7.580-7.620 (d, J= $16 \mathrm{~Hz} 1 \mathrm{H}$, ethylenic), 9.003 $(\mathrm{s}, 1 \mathrm{H},-\mathrm{NH}) ; \mathrm{HRMS}=344.1437[\mathrm{M}+\mathrm{H}]$.

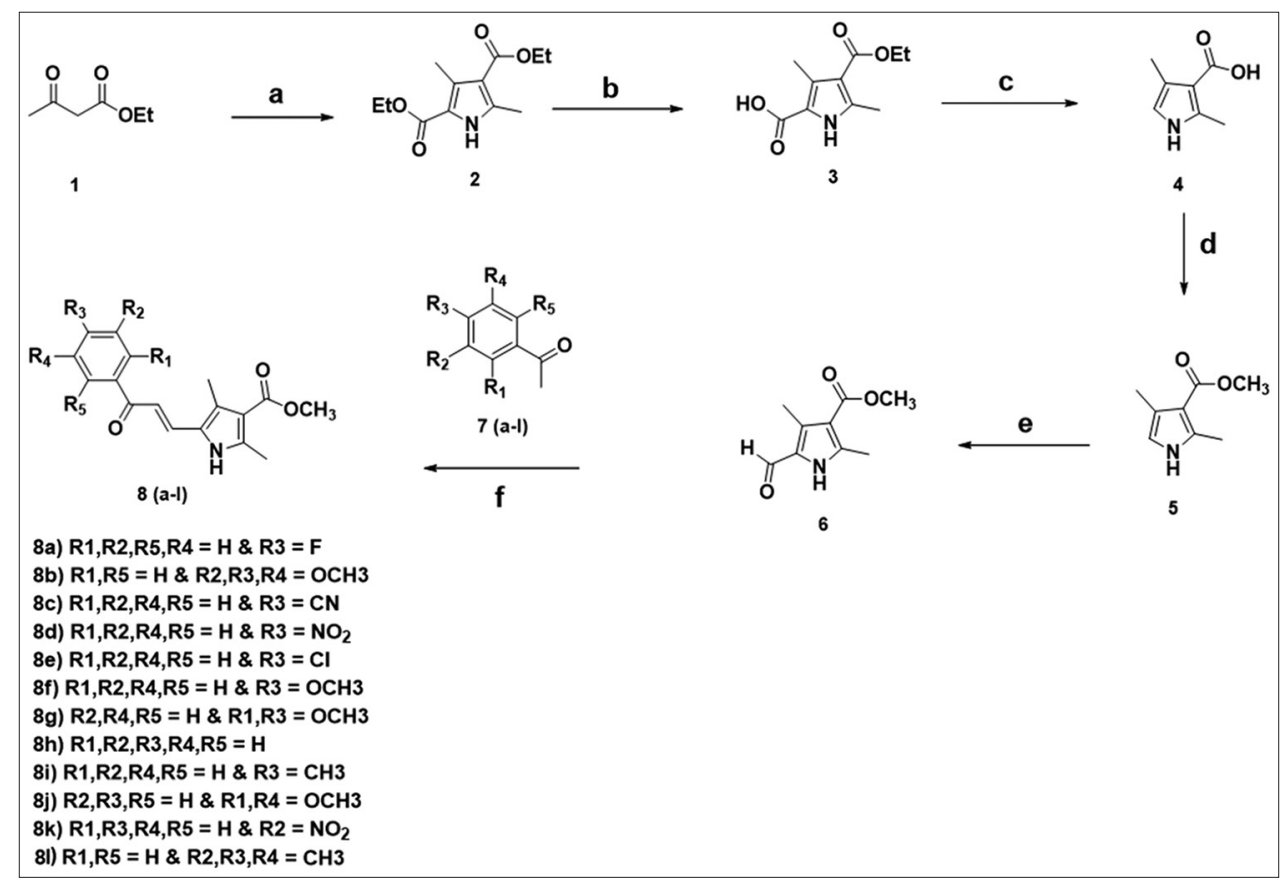

Scheme 1: Synthetic scheme, reagent, and conditions: (a) (i) $\mathrm{NaNO}_{2^{\prime}}$ (ii) $\mathrm{AcOH}, \mathrm{Zn}, 90-95^{\circ} \mathrm{C}$, (b) Aq. $\mathrm{KOH}, \mathrm{EtOH}, 50-60^{\circ} \mathrm{C}$, (c) (i) $\mathrm{HCl}, \mathrm{MeOH}^{\circ}$ 75-80 ${ }^{\circ} \mathrm{C}$, (ii) Aq. $\mathrm{KOH}, \mathrm{MeOH}, 60-65^{\circ} \mathrm{C}$, (d) $\mathrm{Cat}_{2} \mathrm{H}_{2} \mathrm{SO}_{4}, \mathrm{MeOH}, 60-65^{\circ} \mathrm{C}$, (e) DMF/POCl${ }_{3}$, DCM, reflux, (f) Aq.KOH, $\mathrm{MeOH}$, r.t. 
(E)-methyl 2,4-dimethyl-5-(3-(3-nitrophenyl)-3-oxoprop-1-en-1yl)-1H-pyrrole-3-carboxylate (8k)

Yield: 57\%; MF: $\mathrm{C}_{17} \mathrm{H}_{16} \mathrm{~N}_{2} \mathrm{O}_{5} / 328.31$; yellow solid; MP.102-105 ${ }^{\circ} \mathrm{C}$; IR $(\mathrm{KBr}) \mathrm{cm}^{-1}: 3218(-\mathrm{NH}), 1687(\mathrm{C}=0), 1558(\mathrm{C}=\mathrm{C}) ;{ }^{1} \mathrm{H}$ NMR $(400 \mathrm{MHz}$, DMSO- $\left.d_{6}\right) \delta 2.446\left(\mathrm{~s}, 3 \mathrm{H},-\mathrm{CH}_{3}\right), 2.632\left(\mathrm{~s}, 3 \mathrm{H},-\mathrm{CH}_{3}\right), 3.855\left(\mathrm{~s}, 3 \mathrm{H},-\mathrm{OCH}_{3}\right)$, 6.959-6.997 (d, $J=15.2 \mathrm{H}_{\mathrm{z}} 1 \mathrm{H}$, ethylenic), 7.688-7.728 (m, 1H, -Ar), 7.898-7.936 (d, $J=15.2 \mathrm{H}_{\mathrm{z}} 1 \mathrm{H}$, ethylenic), 8.357-8.438 (m, 3H, -Ar), 8.817 (s, 1H, -NH).

(E)-methyl 2,4-dimethyl-5-(3-oxo-3-(3,4,5-trimethylphenyl)prop1-en-1-yl)-1H-pyrrole-3-carboxylate (8l)

Yield: 55\%; MF: $\mathrm{C}_{20} \mathrm{H}_{23} \mathrm{NO}_{3} / 325.40$; yellow solid; MP. $147-150^{\circ} \mathrm{C}$; IR (KBr) cm ${ }^{-1}: 3195(-\mathrm{NH}), 2985(-\mathrm{CH}), 1693(\mathrm{C}=0), 1539$ (C=C); ${ }^{1} \mathrm{H}$ NMR (400 MHz), DMSO- $\left.d_{6}\right) \delta 2.404\left(\mathrm{~s}, 6 \mathrm{H},-\mathrm{CH}_{3}\right), 2.563\left(\mathrm{~s}, 9 \mathrm{H},-\mathrm{CH}_{3}\right)$, $3.831\left(\mathrm{~s}, 3 \mathrm{H},-\mathrm{OCH}_{3}\right.$ ), 7.056-7.095 (d, $J=15.6 \mathrm{~Hz} 1 \mathrm{H}$, ethylenic), 7.2127.232 (s, 1H, -Ar), 7.784-7.823(d, $J=15.6 \mathrm{~Hz} 1 \mathrm{H}$, ethylenic), 7.8617.881(s, 1H, -Ar); 9.269 (s, 1H, -NH).

\section{Biological evaluation}

\section{Antibacterial and antifungal activity}

Some of the synthesized compounds were screened for in vitro antibacterial and antifungal activities against Gram-positive and Gram-negative bacteria. In Gram-positive bacteria, Micrococcus luteus, Bacillus megaterium, Staphylococcus aureus, and Bacillus cereus were used and in Gram-negative bacteria, Salmonella typhi, E. coli, Pseudomonas aeruginosa, and Salmonella abony were used against standard tetracycline. In antifungal activity C. albicans, Saccharomyces cerevisiae and Aspergillus niger were used against standard nystatin. Antimicrobial activities were carried out on nutrient agar with standard composition and by standard procedure of paper disk method [31].

Petri dishes and necessary glassware's were sterilized in hot air oven $\left(190^{\circ} \mathrm{C}, 45 \mathrm{~min}\right)$. The nutrient agar and saline $(0.82 \% \mathrm{NaCl})$ were sterilized in an autoclave $\left(121^{\circ} \mathrm{C}, 15 \mathrm{psi}\right.$, and $\left.20 \mathrm{~min}\right)$. The inoculum was prepared in sterile saline, and optical density of all pathogens was adjusted to 0.10 at $625 \mathrm{~nm}$ on Chemito Spectra scan UV 2600 spectrometer (Mumbai, India), which is equivalent to 0.5 McFarland standards. The nutrient agar plates were prepared by pour plate method [31]. The sensitivity of compounds was tested by the disk diffusion method (paper disk method). All the bacterial cells were cultured in nutrient plates and the compounds to be tested were dissolved in DMSO solvent and were soaked in paper disks. The disks were placed into the plates and incubated at $37^{\circ} \mathrm{C}$ for $24 \mathrm{~h}$. The diameter in millimeter of the zone of inhibition around each disk was measured by the scale.

\section{RESULTS AND DISCUSSION}

\section{Chemistry}

The synthesis of (E)-methyl 2,4-dimethyl-5-(3-oxo-3-phenylprop-1-en1-yl)-1H-pyrrole-3-carboxylate derivatives (8a-8l) Scheme 1. Diethyl 3,5-dimethyl-1H-pyrrole-2,4-dicarboxylate (2) was first obtained through Knorr reaction from acetyl acetate ethyl ester (1) and zinc and acetic acid. The diethyl 3,5-dimethyl-1H-pyrrole-2,4-dicarboxylate (2) has selectively hydrolyzed by $\mathrm{KOH}$ in ethyl alcohol to yield 4-(ethoxycarbonyl)-3,5dimethyl-1H-pyrrole-2-carboxylic acid (3). The 4-(ethoxycarbonyl)3,5-dimethyl-1H-pyrrole-2-carboxylic acid was further subjected to decarboxylation reaction in the presence of hydrochloric acid in methanol and which on further hydrolysis in the presence of potassium hydroxide yield 2,4-dimethyl-1H-pyrrole-3-carboxylic acid compound (4). Compound 4 on esterification reaction using $\mathrm{H}_{2} \mathrm{SO}_{4}$ and methanol to form methyl 2,4-dimethyl-1H-pyrrole-3-carboxylate (5). Compound 5 further subjected to Vilsmeier-Haack formylation reaction to afford methyl 5-formyl-2,4-dimethyl-1H-pyrrole-3-carboxylate (6). The methyl 5-formyl-2,4-dimethyl-1H-pyrrole-3-carboxylate was subjected to base-catalyzed Claisen-Schmidt condensation reaction [32], with appropriate acetophenones (7a-l) in Aq. $\mathrm{KOH}$ in $\mathrm{MeOH}$ to yield (E)-methyl 2,4-dimethyl-5-(3-oxo-3-phenylprop-1-en-1-yl)-1H-pyrrole-

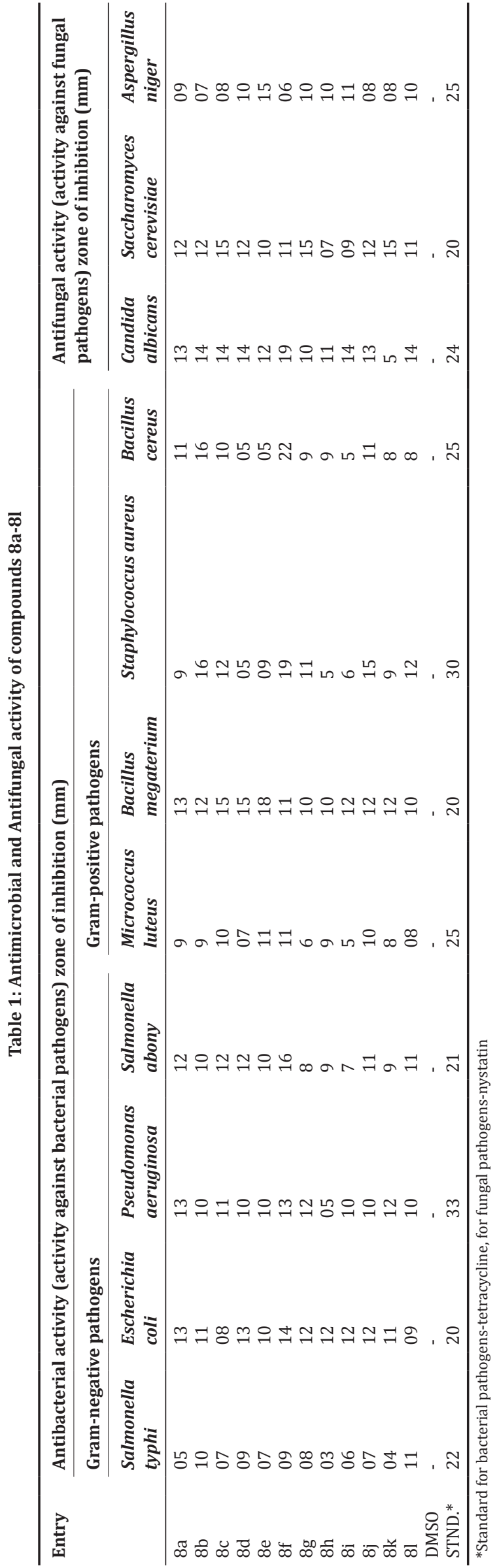


3-carboxylate derivatives (8a-l). The purity of compounds was checked by TLC. Analytical and spectral data ( ${ }^{1} \mathrm{HNMR}$, IR, mass) of newly synthesized compounds were in full agreement with the proposed structure. The structure of 6 is interpreted from spectroscopic data its NMR spectrum exhibit characteristics of the aldehydic proton at $\delta=9.6 \mathrm{ppm}$ and $-\mathrm{NH}$ pyrrole peaks at $\delta=9.9 \mathrm{ppm}$. The structure of $8 \mathrm{a}$ is interpreted from spectroscopic data. The IR spectrum of 8 a shows characteristic bands at 1696 due to $\mathrm{C}=0$ stretching. Its ${ }^{1} \mathrm{H}$ NMR spectrum exhibits the presence of olefinic protons as a doublet at $\delta=7.0$ and 7.8 regions with a mutual coupling constant value $U=15.2$ and $15.6 \mathrm{~Hz}$ ). These observed coupling constant values indicate the presence of $E$ - configuration from the structure and the remaining protons as well aliphatic protons appear at their respective position. The - $\mathrm{NH}$ is highly deshielded and appears at $\delta=9.01 \mathrm{ppm}$. Mass spectrum of $(E)$-methyl 5-(3-(4-fluorophenyl)3-oxoprop-1-en-1-yl)-2,4-dimethyl-1H-pyrrole-3- carboxylate showed $(\mathrm{M}+\mathrm{H})$ peak at 302.11

\section{Antimicrobial activity}

Antimicrobial activity result of synthesized(E)-methyl 2,4-dimethyl-5(3-oxo-3-phenylprop-1-en-1-yl)-1H-pyrrole-3-carboxylate (8a-l) reveals that the compounds $8 \mathrm{f}, 8 \mathrm{e}$, and $8 \mathrm{c}$ were found to possess significant activity while $8 \mathrm{a}, 8 \mathrm{~d}, 8 \mathrm{~g}$, and $8 \mathrm{k}$ showed moderate to good activity compared to the standard reference nystatin and tetracycline Table 1.

The diameter in millimeter of the zone of inhibition around each disk was measured by a scale, and the observed data of antimicrobial and antifungal activities of compounds and the standard drugs are given in Table 1. Among all the compound screened 8f, 8e, and 8c showed significant antibacterial activity against Gram-positive bacteria.

Solvent used-DMSO-The effect of DMSO on pathogen was also checked, but it was found that DMSO does not affect the pathogen.

The concentration of compounds used for the test $-1 \mathrm{mg} / \mathrm{ml}$.

All results (zone of inhibition) are recorded in millimeter (mm).

Compounds $8 \mathrm{a}$ and $8 \mathrm{f}$ showed significant activity against Gram-negative bacteria as comparable with that of standard drug tested. Compounds $8 \mathrm{c}, 8 \mathrm{e}, 8 \mathrm{f}, 8 \mathrm{~g}$, and $8 \mathrm{k}$ showed good activity against fungal pathogens as compared with that of standard drug tested. Hence, the result of all preliminary studies indicated that the substituted $(E)$-methyl 5-(3-(4-methoxyphenyl)-3-oxoprop-1-en-1-yl)-2,4-dimethyl-1Hpyrrole-3-carboxylate (8f), (E)-methyl5-(3-(4-cyanophenyl)-3oxoprop-1-en-1-yl)-2,4-dimethyl-1H-pyrrole-3-carboxylate(8c) and (E)-methyl 5-(3-(4-chlorophenyl)-3-oxoprop-1-en-1-yl)-2,4-dimethyl$1 \mathrm{H}$-pyrrole-3-carboxylate (8e) moiety represent a new class of pharmacophore for broad spectrum of antimicrobial activity.

\section{CONCLUSION}

In summary, we have synthesized a series of pyrrole incorporated new (E)-methyl 2,4-dimethyl-5-(3-oxo-3-phenylprop-1-en-1-yl)$1 \mathrm{H}$-pyrrole-3-carboxylate derivatives(8a-l), and their antimicrobial activities have been evaluated. The compounds $8 \mathrm{f}, 8 \mathrm{e}$, and $8 \mathrm{c}$ demonstrated significant inhibition and $8 \mathrm{a}, 8 \mathrm{~d}, 8 \mathrm{~g}$, and $8 \mathrm{k}$ showed moderate to good activity against the tested strains. The importance of such work lies in the possibilities that the new compounds might be more efficacious drugs against bacteria, which could be helpful in designing more potent antibacterial agent for therapeutic use.

\section{ACKNOWLEDGMENTS}

We are very thankful to the School of Chemical Sciences, Solapur University, Solapur, for providing laboratory facility and thankful to the Department of Microbiology, Dr. Babasaheb Ambedkar Marathwada University, Sub campus, Osmanabad for antimicrobial activity determinations. We are also thankful to the Instrumentation Department of Solapur University for providing us the wonderful facility of spectral analysis.

\section{AUTHORS' CONTRIBUTIONS}

Mahesh Hublikar carried out the experiment, synthesis, and wrote this article. Raghunath Bhosale provided guidance, critical review, and revision. Prashant Dixit supported for biological evaluation of synthesized compounds. Vikas Kadu contributed to the final manuscript. Sachin Shirame contributed for interpretation of data. Dattatraya Raut encouraged Mahesh Hublikar to explore the finding of work.

\section{CONFLICTS OF INTEREST}

The authors declare that they have no conflicts of interest.

\section{REFERENCES}

1. Gholap SS. Pyrrole: An emerging scaffold for construction of valuable therapeutic agents. Eur J Med Chem 2016;110:13-31.

2. Vaya J, Belinky PA, Aviram M. Antioxidant constituents from licorice roots: Isolation, structure elucidation and antioxidative capacity toward LDL oxidation. Free Radic Biol Med 1997;23:302-13.

3. Patole SS, Rajput SS. Synthesis characterization and biological evaluation of (3z, 4z)-3, 4-Bis (substituted phenyl benzylidine)-1(4-Substituted phenyl) pyrrolidine-2,5-dione. Int J Pharm Pharm Sci 2016;8:289-91.

4. Ritter M, Martins RM, Dias D, Pereira CM. Recent advances on the synthesis of chalcones with antimicrobial activities: A brief review. Lett Org Chem 2014;11:498-508.

5. Budhiraja A, Kadian K, Kaur M, Aggarwal V, Garg A, Sapra S, et al. Synthesis and biological evaluation of naphthalene, furan, and pyrrole based chalcones as cytotoxic and antimicrobial agents. Med Chem Res 2012;21:2140.

6. Nowakowska Z. A review of anti-infective and anti-inflammatory chalcones. Eur J Med Chem 2007;42:125-37.

7. Wang YH, Dong HH, Zhao F, Wang J, Yan F, Jiang YY, et al. The synthesis and synergistic antifungal effects of chalcones against drug resistant Candida albicans. Bioorg Med Chem Lett 2016;26:3098-102.

8. Sashidhara KV, Rao KB, Kushwaha P, Modukuri RK, Singh P, Soni I, et al. Novel chalcone-thiazole hybrids as potent inhibitors of drug resistant Staphylococcus aureus. ACS Med Chem Lett 2015;6:809-13.

9. Özdemir A, Altıntop MD, Cantürk Z, Kaplancıklı ZA. Synthesis and in vitro evaluation of furan-based chalcone derivatives as antimicrobial agents. Lett Drug Des Discov 2015;12:607-11.

10. López SN, Castelli MV, Zacchino SA, Domínguez JN, Lobo G, CharrisCharris $\mathrm{J}$, et al. In vitro antifungal evaluation and structure-activity relationships of a new series of chalcone derivatives and synthetic analogues, with inhibitory properties against polymers of the fungal cell wall. Bioorg Med Chem 2001;9:1999-2013.

11. Vibhute YB, Basser MA. Synthesis and activity of a new series of chalcones as antibacterial agents. Indian J Chem 2003;42B:202.

12. Azad M, Muawar MA, Siddiqui HL. Antimicrobial activity and synthesis of quinoline based chalcones. J Appl Sci 2007;7:2485-9.

13. Dinkova-Kostova AT, Massiah MA, Bozak RE, Hicks RJ, Talalay P. Potency of Michael reaction acceptors as inducers of enzymes that protect against carcinogenesis depends on their reactivity with sulfhydryl groups. Proc Natl Acad Sci U S A 2001;98:3404-9.

14. Rezk BM, Haenen GR, van der Vijgh WJ, Bast A. The antioxidant activity of phloretin: The disclosure of a new antioxidant pharmacophore in flavonoids. Biochem Biophys Res Commun 2002;295:9-13.

15. Boeck P, Bandeira Falcão CA, Leal PC, Yunes RA, Filho VC, Torres-Santos EC, et al. Synthesis of chalcone analogues with increased antileishmanial activity. Bioorg Med Chem 2006;14:1538-45.

16. Lawrence NJ, Patterson RP, Ooi LL, Cook D, Ducki S. Effects of alpha-substitutions on structure and biological activity of anticancer chalcones. Bioorg Med Chem Lett 2006;16:5844-8.

17. Nam NH, Kim Y, You YJ, Hong DH, Kim HM, Ahn BZ, et al. Cytotoxic 2',5'-dihydroxychalcones with unexpected antiangiogenic activity. Eur J Med Chem 2003;38:179-87.

18. Won SJ, Liu CT, Tsao LT, Weng JR, Ko HH, Wang JP, et al. Synthetic chalcones as potential anti-inflammatory and cancer chemopreventive agents. Eur J Med Chem 2005;40:103-12

19. Laskin DL, Pendino KJ. Macrophages and inflammatory mediators in tissue injury. Annu Rev Pharmacol Toxicol 1995;35:655-77.

20. Pandey VK, Gupta VD, Tiwari DN. Synthesis of substituted benzoxazines as potential antiviral agents. Indian J Heterocycl Chem 2004;13:399.

21. Edwards ML, Stemerick DM, Sunkara PS. Chalcones: A new class of 
antimitotic agents. J Med Chem 1990;33:1948-54.

22. Bhat BA, Dhar KL, Puri SC, Saxena AK, Shanmugavel M, Qazi GN, et al. Synthesis and biological evaluation of chalcones and their derived pyrazoles as potential cytotoxic agents. Bioorg Med Chem Lett 2005; $15: 3177-80$.

23. Mukherjee S, Kumar V, Prasad AK, Raj HG, Bracke ME, Olsen CE, et al. Synthetic and biological activity evaluation studies on novel 1,3-diaryl propenones. Bioorg Med Chem Lett 2001;9:337-45.

24. Modzelewska A, Pettit C, Achanta G, Davidson NE, Huang P, Khan SR, et al. Anticancer activities of novel chalcone and bis-chalcone derivatives. Bioorg Med Chem 2006;14:3491-5.

25. Calliste CA, Le Bail JC, Trouillas P, Pouget C, Habrioux G, Chulia AJ, et al. Chalcones: Structural requirements for antioxidant, estrogenic and antiproliferative activities. Anticancer Res 2001;21:3949-56.

26. Garg S, Raghav N. Synthesis of novel chalcones of Schiff's bases and to study their effect on bovine serum albumin. Int J Pharm Pharm Sci 2013;6:181-4
27. Hamid SJ, Kubba AA. Synthesis and characterization of new coumarin derivatives containing various moieties with antibacterial activities. Int J Pharm Pharm Sci 2015;7:70-4.

28. Nerule MN, Gaidhane MK, Gaidhane PK. Antioxidant and pharmacological active microwave mediated synthesis of 2-(4/- phenothiazinyl pyrazolyl) pyrroles. Int J Curr Pharm Res 2015;10:29-34.

29. Cooney JV, Beal EJ, Hazlett RN. The synthesis of knorr's pyrrole by inverse addition. Org Prep Proced Int 1983;15:292-5.

30. Liu B, Lin R, Liao JY, Li ZC, et al.Synthesis of Sunitinib. Chin J Pharm 2007;38:539.

31. Kilic A, Baysallar M, Besirbellioglu B, Salih B, Sorkun K, Tanyuksel M. In vitro antimicrobial activity of propolis against methicillin-resistant Staphylococcus aureus and vancomycin resistant Enterococcus faecium. Ann Microbiol 2005;55:113.

32. Godkar PB. Text Book of Medicinal Laboratory Technology. Bombay, India: Bhalani Publishing House; 1996. 